\title{
Rate of Convergence of Hermite-Fejér Interpolation on the Unit Circle
}

\author{
E. Berriochoa, ${ }^{1}$ A. Cachafeiro, ${ }^{2}{ }^{\text {J. Díaz, }},{ }^{2}$ and E. Martínez-Brey ${ }^{2}$ \\ ${ }^{1}$ Departamento de Matemática Aplicada I, Facultad de Ciencias, Universidad de Vigo, 32004 Ourense, Spain \\ ${ }^{2}$ Departamento de Matemática Aplicada I, E. Ingeniería Industrial, Universidad de Vigo, 36310 Vigo, Spain
}

Correspondence should be addressed to E. Berriochoa; esnaola@uvigo.es

Received 13 November 2012; Revised 2 March 2013; Accepted 4 March 2013

Academic Editor: Roberto Barrio

Copyright (C) 2013 E. Berriochoa et al. This is an open access article distributed under the Creative Commons Attribution License, which permits unrestricted use, distribution, and reproduction in any medium, provided the original work is properly cited.

The paper deals with the order of convergence of the Laurent polynomials of Hermite-Fejér interpolation on the unit circle with nodal system, the $n$ roots of a complex number with modulus one. The supremum norm of the error of interpolation is obtained for analytic functions as well as the corresponding asymptotic constants.

\section{Introduction}

The paper is devoted to study the Hermite-Fejér interpolation problem on the unit circle $\mathbb{T}$. This topic has attracted the interest of many researchers in recent years, and it has been the subject of several studies. In [1] Fejér's classical result is extended to the unit circle. It is well known that it ensures uniform convergence of Hermite-Fejér interpolants to continuous functions on $[-1,1]$ taking as nodal system the Chebyshev points (see [2-4]). Specifically, in [1] the authors consider the nodal system of the $n$ roots of a complex number with modulus one. Then it is proved that the Laurent polynomials of Hermite-Fejér interpolation for a given continuous function $f$ on the unit circle uniformly converge to $f$.

In [5] second Fejér's theorem concerning the Hermite interpolation with nonvanishing derivatives is extended, to the unit circle. New conditions for the derivatives are obtained in order that the Hermite interpolants uniformly converge to continuous functions on the unit circle.

An algorithm for efficient computing of the coefficients of the Laurent polynomials of Hermite-Fejér and Hermite interpolation with equally spaced nodes on the unit circle was given in [6]. These results were extended to the bounded interval, and the corresponding expressions can be evaluated using the techniques given in [7]. Some results concerning the convergence were obtained in [8]. The convergence of the Laurent polynomials of Hermite-Fejér interpolation has been studied in [8] for analytic functions defined on open sets containing the unit disk. The results describe the behavior outside and inside the unit disk and are extended to the case of Hermite interpolation, that is, with nonvanishing derivatives.

In the case of bounded interval, the supremum norm of the error of interpolation was studied in several papers (see [9]). In particular a lower bound for the order of convergence of Hermite-Fejér interpolation was obtained in [10] for general nodal systems. Now, in the present paper, we study the same problem for the error of interpolation on the unit circle by taking into account the results obtained in [8].

The organization of the paper is as follows. Section 2 is dedicated to obtain the results for the order of convergence for Laurent polynomials, in other words to the polynomial case. Section 3 contains the extension of the preceding results for analytic functions. The order of convergence and the asymptotic constants are deduced in our main result for analytic functions on an open disk containing the unit circle. As a consequence, the result is generalized to analytic functions outside an open disk with radius less than one, and it is also generalized, in Section 4, to analytic functions on an open annulus containing the unit circle. Finally, the last section is devoted to some numerical experiments to reveal the contributions of our results. 


\section{The Polynomial Case}

Let $\left\{\alpha_{j}\right\}_{j=0}^{n-1}$ be the $n$ roots of a complex number $\lambda$ with modulus 1 . We recall that the Hermite interpolation problem on the unit circle $\mathbb{W}$ with nodal system $\left\{\alpha_{j}\right\}_{j=0}^{n-1}$ consists in obtaining a Laurent polynomial $H_{-(n-1), n}(z)$ of $\Lambda_{-(n-1), n}[z]=$ $\operatorname{span}\left\{z^{k}:-(n-1) \leq k \leq n\right\}$ that satisfies the following interpolation conditions:

$$
\begin{array}{r}
H_{-(n-1), n}\left(\alpha_{j}\right)=u_{j}, \quad H_{-(n-1), n}^{\prime}\left(\alpha_{j}\right)=v_{j}, \\
j=0, \ldots, n-1,
\end{array}
$$

where $\left\{u_{j}\right\}_{j=0}^{n-1}$ and $\left\{v_{j}\right\}_{j=0}^{n-1}$ are sets of fixed complex numbers.

The particular case when $v_{j}=0$ for all $j$ is called the Hermite-Fejér interpolation problem, and the corresponding interpolation polynomial is denoted by $\mathrm{HF}_{-(n-1), n}(z)$. When $u_{j}=f\left(\alpha_{j}\right),(0 \leq j \leq n-1)$, for a given function $f(z)$ defined on $\mathbb{T}$, we denote the Hermite-Fejér interpolation polynomial by $H F_{-(n-1), n}(f(z), z)$. To estimate the interpolation error between $f(z)$ and $H F_{-(n-1), n}(f(z), z)$ we consider their difference that we denote by

$$
\Delta_{n}(f(z), z)=f(z)-H F_{-(n-1), n}(f(z), z) .
$$

It is well known that $H F_{-(n-1), n}(f(z), z)$ can be computed in terms of the fundamental polynomial of Hermite interpolation, $A_{j}(z)$, as follows:

$$
H F_{-(n-1), n}(f(z), z)=\sum_{j=0}^{n-1} f\left(\alpha_{j}\right) A_{j}(z),
$$

where $A_{j}(z)$ is given by $A_{j}(z)=\alpha_{j}^{n+1}\left(z^{n}-\lambda\right)^{2} / z^{n-1} n^{2}$ $\lambda^{2}\left(z-\alpha_{j}\right)^{2}$ and it holds that

$$
\left|A_{j}(z)\right|=\frac{1}{n^{2}} \frac{\left|\left(z^{n}-\lambda\right)^{2}\right|}{\left|\left(z-\alpha_{j}\right)^{2}\right|} \leq 1 \quad \text { on } \mathbb{T} .
$$

Representation (3) can be seen in [1], and (4) can be seen in [5].

We recall that for a continuous function $f(z)$ defined on $\mathbb{T}, H F_{-(n-1), n}(f(z), z)$ converges to $f(z)$ uniformly on $\mathbb{T}$, as it can be seen in [1].

These results can be improved in case of polynomial functions. Indeed we can obtain nice explicit expressions for $H F_{-(n-1), n}(f(z), z)$ and $\Delta_{n}(f(z), z)$ for the polynomial case; that is, in this section we are going to use an algebraic polynomial or a Laurent polynomial in the role of $f(z)$.

Theorem 1. Let $k$ be a fixed positive integer number. For $n>k$ the following conditions hold that

(i) $H F_{-(n-1), n}\left(z^{k}, z\right)=(1-k / n) z^{k}+(k \lambda / n) z^{k-n}$;

(ii) $\Delta_{n}\left(z^{k}, z\right)=(k / n) z^{k}\left(1-\lambda / z^{n}\right)$;

(iii) $H_{-(n-1), n}\left(z^{k}, z\right)$ converges to $z^{k}$ uniformly on compact subsets $K$ of $0<|z| \geq 1$ with order of convergence $O\left(n^{-1}\right)$; (iv) $H F_{-(n-1), n}\left(z^{-k}, z\right)=(1-k / n) z^{-k}+(k / n \lambda) z^{-k+n}$;

(v) $\Delta_{n}\left(z^{-k}, z\right)=(-k / n) z^{-k}\left(z^{n} / \lambda-1\right)$;

(vi) $H_{-(n-1), n}\left(z^{-k}, z\right)$ converges to $z^{-k}$ uniformly on compact subsets $K$ of $0<|z| \leq 1$ with order of convergence $O\left(n^{-1}\right)$.

Proof. In order to obtain (i), take into account that when we evaluate the proposed Laurent polynomial $(1-k / n) z^{k}+$ $(k \lambda / n) z^{k-n}$ at $\alpha_{j}$ we have

$$
\left(\frac{n-k}{n}\right) \alpha_{j}^{k}+\frac{k \lambda}{n} \alpha_{j}^{k-n}=\left(\frac{n-k}{n}\right) \alpha_{j}^{k}+\frac{k \lambda}{n \lambda} \alpha_{j}^{k}=\alpha_{j}^{k},
$$

that is, the interpolation conditions for the function are fulfilled. In the same way, when we evaluate the corresponding derivative at $\alpha_{j}$ we obtain

$$
\begin{aligned}
& \left(\frac{n-k}{n}\right) k \alpha_{j}^{k-1}+\frac{k \lambda}{n}(k-n) \alpha_{j}^{k-n-1} \\
& \quad=\left(\frac{n-k}{n}\right) k \alpha_{j}^{k-1}+\frac{k \lambda}{n \lambda}(k-n) \alpha_{j}^{k-1}=0 .
\end{aligned}
$$

Thus the existence and uniqueness of the Hermite interpolation polynomial ensures (i).

(ii) It is an immediate consequence of (i) and the definition of $\Delta_{n}\left(z^{k}, z\right)$.

(iii) Take into account that

$$
\left|n \Delta_{n}\left(z^{k}, z\right)\right|=k\left|z^{k}\left(1-\frac{\lambda}{z^{n}}\right)\right|
$$

where the last expression is uniformly bounded if $z \in K$ and $n$ is large enough.

(iv), (v), and (vi) can be proved proceeding in the same way.

Remark 2. The resulting expressions for $\Delta_{n}\left(z^{k}, z\right)$ and $\Delta_{n}\left(z^{-k}, z\right)$, given in the preceding theorem, can be rewritten as follows:

$$
\begin{gathered}
\Delta_{n}\left(z^{k}, z\right)=\frac{1}{n} z\left(\frac{z^{n}-\lambda}{z^{n}}\right)\left(z^{k}\right)^{\prime}, \\
\Delta_{n}\left(z^{-k}, z\right)=\frac{1}{n} z\left(\frac{z^{n}-\lambda}{\lambda}\right)\left(z^{-k}\right)^{\prime} .
\end{gathered}
$$

\section{Corollary 3. The following hold.}

(i) If $p_{1}(z)$ is a Laurent polynomial with nonnegative powers of $z$, that is, $p_{1}(z)$ is an algebraic polynomial, then

(a) $\Delta_{n}\left(p_{1}(z), z\right)=\frac{1}{n} z\left(\left(z^{n}-\lambda\right) / z^{n}\right) p_{1}^{\prime}(z)$.

(b) If $K$ is a compact subset of $0<|z|<1$, then

$$
\lim _{n \rightarrow \infty}\left\|n \Delta_{n}\left(p_{1}(z), z\right)\right\|_{\infty, K}=\max _{z \in K}\left|z p_{1}^{\prime}(z)\right|,
$$


(c) If $K$ is a compact subset of $|z|=1$ with no isolated points, then

$$
\lim _{n \rightarrow \infty}\left\|n \Delta_{n}\left(p_{1}(z), z\right)\right\|_{\infty, K}=2 \max _{z \in K}\left|p_{1}^{\prime}(z)\right| .
$$

(ii) If $p_{2}(z)$ is a Laurent polynomial with only negative powers of $z$, then

(a) $\Delta_{n}\left(p_{2}(z), z\right)=1 / n z\left(\left(z^{n}-\lambda\right) / \lambda\right) p_{2}^{\prime}(z)$.

(b) If $K$ is a compact subset of $|z|<1$, then

$$
\lim _{n \rightarrow \infty}\left\|n \Delta_{n}\left(p_{2}(z), z\right)\right\|_{\infty, K}=\max _{z \in K}\left|z p_{2}^{\prime}(z)\right| \text {. }
$$

(c) If $K$ is a compact subset of $|z|=1$ with no isolated points, then

$$
\lim _{n \rightarrow \infty}\left\|n \Delta_{n}\left(p_{2}(z), z\right)\right\|_{\infty, K}=2 \max _{z \in K}\left|p_{2}^{\prime}(z)\right| .
$$

Proof. (i) (a) It is a straightforward consequence of the previous remark.

(i) (b) First of all take into account that for each $z \in K$ it holds that

$$
\lim _{n \rightarrow \infty}\left|n \Delta_{n}\left(p_{1}(z), z\right)\right|=\left|z p_{1}^{\prime}(z)\right|
$$

Therefore, if $\max _{z \in K}\left|z p_{1}^{\prime}(z)\right|$ is attained at $z_{0} \in K$, then for each $z \in K$ the following relation holds:

$$
\lim _{n \rightarrow \infty}\left|\Delta_{n}\left(p_{1}(z), z\right)\right|=\left|z p_{1}^{\prime}(z)\right| \leq\left|z_{0} p_{1}^{\prime}\left(z_{0}\right)\right| \text {. }
$$

On the other hand, since for $z_{0}$ we have $\lim _{n \rightarrow \infty}$ $\left|n \Delta_{n}\left(p_{1}(z), z_{0}\right)\right|=\left|z_{0} p_{1}^{\prime}\left(z_{0}\right)\right|$, then we obtain the result.

(i) (c) If $z_{0}$ is the point where $\max _{z \in K}\left|p_{1}^{\prime}(z)\right|$ is attained, then for each $z \in K \subset \mathbb{T}$ it holds that

$$
\begin{aligned}
\left|n \Delta_{n}\left(p_{1}(z), z\right)\right| & =\left|\left(z^{n}-\lambda\right) p_{1}^{\prime}(z)\right| \\
& \leq 2 \max _{z \in K}\left|p_{1}^{\prime}(z)\right|=2\left|p_{1}^{\prime}\left(z_{0}\right)\right|,
\end{aligned}
$$

which implies that

$$
\lim _{n \rightarrow \infty}\left\|n \Delta_{n}\left(p_{1}(z), z\right)\right\|_{\infty, K} \leq 2 \max _{z \in K}\left|p_{1}^{\prime}(z)\right| .
$$

Due to the continuity of $p_{1}^{\prime}(z)$, for each $\epsilon>0$ there exists a neighborhood of $z_{0}, N_{z_{0}}$, such that for $z \in N_{z_{0}}$ it is $\left|p_{1}^{\prime}(z)\right|>$ $\left|p_{1}^{\prime}\left(z_{0}\right)\right|-\epsilon$. On the other hand, for $n$ large enough any arc of $\mathbb{T}$ contains points $z$ with $z^{n}=-\lambda$, and therefore for some $z \in N_{z_{0}}$, with $z^{n}=-\lambda$, we have

$$
\left|n \Delta_{n}\left(p_{1}(z), z\right)\right| \geq 2\left(\left|p_{1}^{\prime}\left(z_{0}\right)\right|-\epsilon\right) .
$$

Then we obtain

$$
\lim _{n \rightarrow \infty}\left\|n \Delta_{n}\left(p_{1}(z), z\right)\right\|_{\infty, K}=2 \max _{z \in K}\left|p_{1}^{\prime}(z)\right| .
$$

To obtain (ii) (a), (b), and (c) proceed in the same way.
Theorem 4. Let $p(z)=p_{1}(z)+p_{2}(z)$ be a Laurent polynomial with positive and negative powers of $z, p_{1}(z)$ and $p_{2}(z)$, respectively. It holds that

(i) $\Delta_{n}(p(z), z)=1 / n z\left(z^{n}-\lambda\right)\left(p_{1}^{\prime}(z) / z^{n}+p_{2}^{\prime}(z) / \lambda\right)$;

(ii) if $K$ is a compact subset of $\mathbb{T}$ with no isolated points, then

$$
\begin{aligned}
\lim _{n \rightarrow \infty} & \left\|n \Delta_{n}(p(z), z)\right\|_{\infty, K} \\
= & \max _{z \in K, \beta \in \mathbb{T}}\left|(\beta-1)\left(p_{1}^{\prime}(z)+\beta p_{2}^{\prime}(z)\right)\right| .
\end{aligned}
$$

Proof. It is clear that $\max _{z \in K, \beta \in \mathbb{T}}\left|(\beta-1)\left(p_{1}^{\prime}(z)+\beta p_{2}^{\prime}(z)\right)\right|$ exists, it is positive, and it is attained at a point $\left(z_{0}, \beta_{0}\right)$. We denote this maximum by $m$. Besides, since $\left(z^{n} / \lambda-1\right)\left(p_{1}^{\prime}(z)+\right.$ $\left.\left(z^{n} / \lambda\right) p_{2}^{\prime}(z)\right)$ can be represented as $(\beta-1)\left(p_{1}^{\prime}(z)+\beta p_{2}^{\prime}(z)\right)$ with $\beta \in \mathbb{T}$, then

$$
\begin{aligned}
\limsup _{n \rightarrow \infty}\left\|n \Delta_{n}(p(z), z)\right\|_{\infty, K} \\
\quad \leq \max _{z \in K, \beta \in \mathbb{T}}\left|(\beta-1)\left(p_{1}^{\prime}(z)+\beta p_{2}^{\prime}(z)\right)\right| .
\end{aligned}
$$

Due to the continuity of $(\beta-1)\left(p_{1}^{\prime}(z)+\beta p_{2}^{\prime}(z)\right)$, for each $\epsilon>0$ there exists a neighborhood of $\left(z_{0}, \beta_{0}\right), N_{\left(z_{0}, \beta_{0}\right)}$, such that for $(z, \beta) \in N_{\left(z_{0}, \beta_{0}\right)}$ it is

$$
\left|(\beta-1)\left(p_{1}^{\prime}(z)+\beta p_{2}^{\prime}(z)\right)\right|>m-\epsilon,
$$

which implies, in particular, $m-\left|\left(\beta_{0}-1\right)\left(p_{1}^{\prime}(z)+\beta_{0} p_{2}^{\prime}(z)\right)\right|<\epsilon$. Moreover, taking into account that for $n$ large enough any arc $A \subset \mathbb{T}$, with $z_{0} \in A$, contains points $z$ with $z^{n} / \lambda=\beta_{0}$, then we have

$$
\left\|n \Delta_{n}(p(z), z)\right\|_{\infty, K} \geq m-\epsilon .
$$

Remark 5. The previous result can be rewritten as follows:

$$
\begin{aligned}
& \left\|\Delta_{n}(p(z), z)\right\|_{\infty, K} \\
& \quad \approx \frac{\max _{z \in K, \beta \in \mathbb{T}}\left|(\beta-1)\left(p_{1}^{\prime}(z)+\beta p_{2}^{\prime}(z)\right)\right|}{n},
\end{aligned}
$$

where $\asymp$ means that the sequences are equivalent.

\section{Rate of Convergence for Analytic Functions on a Disk}

In this section we extend the previous results to analytic functions. Indeed we study the supremum norm of $\Delta_{n}(F(z), z),\left\|\Delta_{n}(F(z), z)\right\|_{\infty, C_{\rho}}$ for analytic functions $F$ on an open disk containing $\mathbb{T}$ along a circumference $C_{\rho}$ of radius $\rho \geq 1$.

Theorem 6. Let $F(z)$ be a nonconstant analytic function defined on an open disk $D(0, R)$, with $R>1$, and let 
$H_{-(n-1), n}(F(z), z)$ be its Hermite-Fejér interpolation polynomial corresponding to the $n$ roots of $\lambda$. If $C_{\rho}$ is the circumference with radius $\rho,(1 \leq \rho<R)$, and \|\|$_{\infty, C_{\rho}}$ is the supremum norm on $C_{\rho}$, then there exist $C_{1}, C_{2}>0$ satisfying

$$
\frac{C_{1}}{n} \leq\left\|F(z)-H F_{-(n-1), n}(F(z), z)\right\|_{\infty, C_{\rho}} \leq \frac{C_{2}}{n} .
$$

Proof. We exclude the constant case because we have $F(z)-$ $H F_{-(n-1), n}(F(z), z)=0$ in this situation. So let $F(z)=$ $\sum_{k=0}^{\infty} a_{k} z^{k}$ be a nonconstant analytic function. Taking into account that the evaluation of $z^{k+l n}$ at $\alpha_{j}$ is $\alpha_{j}^{k+l n}=\alpha_{j}^{k} \alpha_{j}^{l n}=$ $\alpha_{j}^{k} \lambda^{l}$, we have $H F_{-(n-1), n}\left(z^{k+l n}, z\right)=\lambda^{l} H F_{-(n-1), n}\left(z^{k}, z\right)$. Then by using Theorem 1 we can write

$$
\begin{aligned}
F(z)- & H F_{-(n-1), n}(F(z), z) \\
= & \underbrace{\sum_{k=n}^{\infty} a_{k} z^{k}}_{(1)}+\underbrace{\sum_{k=0}^{n-1}\left(\frac{k}{n} a_{k} z^{k}\right)}_{(2)} \\
& +\underbrace{\sum_{k=0}^{n-1}\left(\frac{-k \lambda}{n} a_{k} z^{-(n-k)}\right)}_{(3)}+\underbrace{\sum_{k=0}^{n-1}\left(\sum_{l=1}^{\infty} a_{k+l n} \lambda^{l}\right) \frac{k-n}{n} z^{k}}_{(5)} \\
& +\underbrace{}_{\sum_{k=0}^{n-1}\left(\sum_{l=1}^{\infty} a_{k+l n} \lambda^{l}\right) \frac{-k}{n} z^{-(n-k)}}
\end{aligned}
$$

Let $z$ be a point belonging to $C_{\rho}=\{z:|z|=\rho\}$ with $\rho \geq 1$ and $R_{1}$, such that $\rho<R_{1}<R$. Then there exists a positive constant $K_{1}$, such that for $n$ large enough we have

$$
\begin{gathered}
|(1)|=\left|\sum_{k=n}^{\infty} a_{k} z^{k}\right| \leq \frac{\left(\rho / R_{1}\right)^{n}}{1-\rho / R_{1}} \leq K_{1}\left(\frac{1}{R_{1}}\right)^{n}, \\
|(4)|=\left|\sum_{k=0}^{n-1}\left(\sum_{l=1}^{\infty} a_{k+l n} \lambda^{l}\right) \frac{k-n}{n} z^{k}\right| \leq \frac{\left(1 / R_{1}\right)^{n}}{1-1 / R_{1}} \sum_{k=0}^{n-1}\left(\frac{\rho}{R_{1}}\right)^{k} \\
=\frac{R_{1}}{R_{1}-1} \frac{1}{R_{1}^{n}} \frac{1-\left(\rho / R_{1}\right)^{n}}{1-\rho / R_{1}} \leq K_{1}\left(\frac{1}{R_{1}}\right)^{n}, \\
|(5)| \leq K_{1}\left(\frac{1}{R_{1}}\right)^{n} .
\end{gathered}
$$

Now we consider (2) and (3) as follows:

$$
\begin{aligned}
& |(2)+(3)| \\
& =\left|\sum_{k=0}^{n-1} \frac{k}{n} a_{k} z^{k}+\sum_{k=0}^{n-1} \frac{-k \lambda}{n} a_{k} z^{-(n-k)}\right| \\
& =\left|\frac{z}{n}\left(1-\frac{\lambda}{z^{n}}\right) \sum_{k=0}^{n-1} k a_{k} z^{k-1}\right|
\end{aligned}
$$

$$
\begin{aligned}
& =\left|\frac{z}{n}\left(1-\frac{\lambda}{z^{n}}\right)\left(F^{\prime}(z)-\sum_{k=n}^{\infty} k a_{k} z^{k-1}\right)\right| \\
& \leq \frac{\rho}{n}\left|1-\frac{\lambda}{z^{n}}\right|\left(\left\|F^{\prime}\right\|_{\infty, C_{\rho}}+\sum_{k=n}^{\infty} k \frac{1}{R_{1}^{k}} \rho^{k-1}\right) \\
& \leq \frac{2 \rho}{n}\left(\left\|F^{\prime}\right\|_{\infty, C_{\rho}}+\frac{1}{\rho} \sum_{k=n}^{\infty} k\left(\frac{\rho}{R_{1}}\right)^{k}\right) \\
& =\frac{2 \rho}{n}\left(\left\|F^{\prime}\right\|_{\infty, C_{\rho}}+\frac{1}{\rho} \frac{n\left(\rho / R_{1}\right)^{n}}{1-\rho / R_{1}}+\left(\frac{\rho}{R_{1}}\right)^{n+1} \frac{1}{\left(1-\rho / R_{1}\right)^{2}}\right) .
\end{aligned}
$$

So we obtain that there exists a positive constant $C_{2}$, such that for $n$ large enough

$$
\left\|F(z)-H F_{-(n-1), n}(F(z), z)\right\|_{\infty, C_{\rho}} \leq \frac{C_{2}}{n} .
$$

In order to obtain the lower bound we use the following inequality:

$$
\begin{aligned}
|(2)+(3)|-|(1)|-|(4)|-|(5)| \\
\quad \leq\left|F(z)-H F_{-(n-1), n}(F(z), z)\right| .
\end{aligned}
$$

For an arbitrary $z \in C_{\rho}$ and $n$ large enough we have

$$
-3 K_{1}\left(\frac{1}{R_{1}}\right)^{n} \leq-|(1)|-|(4)|-|(5)| .
$$

On the other hand, as the zeros of $F^{\prime}(z)$ cannot have accumulation points on $D(0, R)$, then for $\epsilon>0$ there exist an $\operatorname{arc} A\left(C_{\rho}\right) \subset C_{\rho}$ and a positive constant $m_{1}=\left\|F^{\prime}\right\|_{\infty, C_{\rho}}$, such that

$$
0<m_{1}-\epsilon \leq\left|F^{\prime}(z)\right| \quad \forall z \in A\left(C_{\rho}\right)
$$

Next we study two different cases $\rho>1$ and $\rho=1$.

(i) If $\rho>1$ it holds that

$$
\begin{array}{r}
\frac{\rho}{n}\left|1-\frac{\lambda}{z^{n}}\right|\left(m_{1}-\epsilon-\left|\sum_{k=n}^{\infty} k a_{k} z^{k-1}\right|\right) \\
\leq|(2)+(3)| \quad \text { for } z \in A\left(C_{\rho}\right)
\end{array}
$$

and, as before, for $n$ large enough there exist positive constants $D$ and $E$, such that for $z \in C_{\rho}$ we have

$$
\left|\sum_{k=n}^{\infty} k a_{k} z^{k-1}\right| \leq \frac{\rho^{n}}{R_{1}^{n}} n D \leq \frac{E}{n} .
$$

Thus for $n$ large enough and $z \in A\left(C_{\rho}\right)$ we have

$$
0<\frac{\rho}{n}\left|1-\frac{\lambda}{z^{n}}\right|\left(m_{1}-\frac{E}{n}\right)<|(2)+(3)| .
$$


Then there exists $M>0$ satisfying

$$
\frac{M}{n}<\frac{\rho}{n}\left|1-\frac{\lambda}{z^{n}}\right|\left(m_{1}-\frac{E}{n}\right)<|(2)+(3)| .
$$

(ii) If $\rho=1$, we consider an $\operatorname{arc} \mathscr{A}\left(C_{\rho}\right) \subset A\left(C_{\rho}\right)$ with $\mid z^{n}-$ $\lambda \mid>m_{2}>0$ for $z \in \mathscr{A}\left(C_{\rho}\right)$ and a positive constant $m_{2}>0$. Then

$$
\left|\frac{z}{n}\left(1-\frac{\lambda}{z^{n}}\right)\right|=\frac{1}{n}\left|\frac{z^{n}-\lambda}{z^{n}}\right|=\frac{1}{n}\left|z^{n}-\lambda\right|>\frac{m_{2}}{n} .
$$

Proceeding in the same way as in the previous case we have $\left(m_{2} / n\right)\left(m_{1}-E / n\right)<|(2)+(3)|$. Thus there exists $M>0$, such that

$$
\frac{M}{n}<|(2)+(3)|
$$

Taking into account (35) and (37) we have for $n$ large enough, $\rho \geq 1$ and $z \in \mathscr{A}\left(C_{\rho}\right)$

$$
\begin{aligned}
\frac{M}{n}- & 3 K_{1}\left(\frac{1}{R_{1}}\right)^{n} \\
& \leq\left|F(z)-H F_{-(n-1), n}(F(z), z)\right| \\
& \leq\left\|F(z)-H F_{-(n-1), n}(F(z), z)\right\|_{\infty, C_{\rho}} .
\end{aligned}
$$

Then it is straightforward that there exists $C_{1}$, such that

$$
\frac{C_{1}}{n}<\left\|F(z)-H F_{-(n-1), n}(F(z), z)\right\|_{\infty, C_{\rho}}
$$

for $n$ large enough.

\section{Remark 7. Notice that}

(i) the constants $C_{1}$ and $C_{2}$ are closely related to the supremum norm $\left\|F^{\prime}\right\|_{\infty, C_{\rho}}$;

(ii) clearly we can obtain an analogous result for nonconstant analytic function defined on $|z|>r$ with $r<1$.

\section{Rate of Convergence for Analytic Functions on an Annulus}

Next we deal with the case of analytic functions on an open annulus containing $\mathbb{T}$. We obtain explicit expressions for $n \Delta_{n}(F(z), z)$ and the asymptotic behavior of its supremum norm; that is, we obtain the order of convergence and the asymptotic constant.

Throughout this section we consider a function $F$ with Laurent expansion at $z=0$ given by $F(z)=\sum_{k=-\infty}^{\infty} a_{k} z^{k}$ which converges on an annulus containing $\mathbb{T}$. Then there exist $C>0$ and $r, 0<r<1$, such that $\left|a_{-k}\right|,\left|a_{k}\right|<C r^{k}$ for every $k \geq 0$.

For each $n \geq 2$ we denote by $P_{n-2}(z)=\sum_{k=0}^{n-2} a_{k} z^{k}$ and by $\widetilde{P}_{n-2}(z)=\sum_{k=n-1}^{\infty} a_{k} z^{k}$. In the same way we denote by $Q_{n-2}(z)=\sum_{k=-(n-2)}^{-1} a_{k} z^{k}$ and by $\widetilde{Q}_{n-2}(z)=$ $\sum_{k=-\infty}^{-(n-1)} a_{k} z^{k}$ (if $n=2, Q_{n-2}(z)=0$ ).

Furthermore we denote by $P(z)=P_{n-2}(z)+\widetilde{P}_{n-2}(z)$ and by $Q(z)=Q_{n-2}(z)+\widetilde{Q}_{n-2}(z)$.

Then we have the following decompositions of $F(z)$ for each $n \geq 2$ :

$$
\begin{aligned}
F(z)= & P(z)+Q(z) \\
= & P_{n-2}(z)+\widetilde{P}_{n-2}(z) \\
& +Q_{n-2}(z)+\widetilde{Q}_{n-2}(z) .
\end{aligned}
$$

By using this notation for the decompositions of $F(z)$ we obtain the following results.

Lemma 8. In our conditions it holds that

$$
\begin{array}{r}
n \Delta_{n}\left(\widetilde{P}_{n-2}(z)+\widetilde{Q}_{n-2}(z), z\right)=o\left(r_{1}^{n}\right) \\
\text { for each } r_{1} \text { with } r<r_{1}<1, z \in \mathbb{T} .
\end{array}
$$

Proof. If $|z|=1$ we have $\left|\widetilde{P}_{n-2}(z)\right| \leq C\left(r^{n-1} /(1-r)\right)$ and $\left|\widetilde{Q}_{n-2}(z)\right| \leq C\left(r^{n-1} /(1-r)\right)$. On the other hand, by using (3) and (4) we have for $z \in \mathbb{T}$

$$
\begin{aligned}
& \left|H F_{-(n-1), n}\left(\widetilde{P}_{n-2}(z), z\right)\right|=\left|\sum_{j=0}^{n-1} \widetilde{P}_{n-2}\left(\alpha_{j}\right) A_{j}(z)\right| \leq n C \frac{r^{n-1}}{1-r}, \\
& \left|H F_{-(n-1), n}\left(\widetilde{Q}_{n-2}(z), z\right)\right|=\left|\sum_{j=0}^{n-1} \widetilde{Q}_{n-2}\left(\alpha_{j}\right) A_{j}(z)\right| \leq n C \frac{r^{n-1}}{1-r} .
\end{aligned}
$$

Then we can write

$$
\begin{aligned}
\left|\Delta_{n}\left(\widetilde{P}_{n-2}(z)+\widetilde{Q}_{n-2}(z), z\right)\right| \\
\leq\left|\widetilde{P}_{n-2}(z)\right|+\left|\widetilde{Q}_{n-2}(z)\right| \\
\quad+\left|H F_{-(n-1), n}\left(\widetilde{P}_{n-2}(z), z\right)\right| \\
\quad+\left|H F_{-(n-1), n}\left(\widetilde{Q}_{n-2}(z), z\right)\right| \\
\leq C\left(\frac{r^{n-1}}{1-r}+\frac{r^{n-1}}{1-r}+n \frac{r^{n-1}}{1-r}+n \frac{r^{n-1}}{1-r}\right)
\end{aligned}
$$

and the result is proved.

Lemma 9. In our conditions the following holds.

(i) For $z \in \mathbb{T}$ and $r_{1}$, such that $r<r_{1}<1$

$$
\begin{aligned}
n \Delta_{n}\left(P_{n-2}(z), z\right) & =z\left(\frac{z^{n}-\lambda}{z^{n}}\right) P_{n-2}^{\prime}(z) \\
& =z\left(\frac{z^{n}-\lambda}{z^{n}}\right) P^{\prime}(z)+o\left(r_{1}^{n}\right) .
\end{aligned}
$$


TABLE 1

\begin{tabular}{lccc}
\hline$p$ & $n$ & Max detected in $K_{1}$ of $\left|n \Delta_{n}(F(z), z)\right| /\left|P^{\prime}(1)-Q^{\prime}(1)\right|$ & Max detected in $K_{2}$ of $\left|n \Delta_{n}(F(z), z)\right| /\left|P^{\prime}(1)-Q^{\prime}(1)\right|$ \\
\hline 4 & 16 & 0.713835 & 1.68672 \\
6 & 64 & 0.939856 & 1.9788 \\
8 & 256 & 0.997746 & 1.99866 \\
10 & 1024 & 0.995921 & 1.99992 \\
12 & 4096 & 0.998066 & 1.99999 \\
14 & 16384 & 1.00795 & 2. \\
\hline
\end{tabular}

TABLE 2

\begin{tabular}{cccc}
\hline$p$ & $n$ & Max detected in $K_{1}$ of $\left|n \Delta_{n}(F(z), z)\right| /\left|P^{\prime}(1)-Q^{\prime}(1)\right|$ & Max detected in $K_{2}$ of $\left|n \Delta_{n}(F(z), z)\right| /\left|P^{\prime}(1)-Q^{\prime}(1)\right|$ \\
\hline 4 & 16 & 1.40634 & 1.90275 \\
6 & 64 & 1.53754 & 1.99369 \\
8 & 256 & 1.56734 & 1.99959 \\
10 & 1024 & 1.57482 & 1.99957 \\
12 & 4096 & 1.57653 & 2. \\
14 & 16384 & 1.57144 & 2. \\
\hline
\end{tabular}

(ii) For $z \in \mathbb{T}$ and $r_{1}$, such that $r<r_{1}<1$

$$
\begin{aligned}
n \Delta_{n}\left(Q_{n-2}(z), z\right) & =z\left(\frac{z^{n}-\lambda}{\lambda}\right) Q_{n-2}^{\prime}(z) \\
& =z\left(\frac{z^{n}-\lambda}{\lambda}\right) Q_{(z)}^{\prime}+o\left(r_{1}^{n}\right) .
\end{aligned}
$$

Proof. (i) Take into account (i) (a) in Corollary 3 in order to prove the first equality. To obtain the second equality take into account that for $|z|=1, P_{n-2}^{\prime} P^{\prime}(z)=o\left(r_{1}\right)^{n}$, and $z\left(\left(z^{n}-\lambda\right) / z^{n}\right)$ is bounded.

(ii) Proceed in the same way.

Theorem 10. In our conditions the following holds.

(i) For $z \in \mathbb{T}$ and $r_{1}$ such that $r<r_{1}<1$

$$
\begin{aligned}
n \Delta_{n}(F(z), z)= & z\left(\frac{z^{n}-\lambda}{z^{n}}\right) P^{\prime}(z) \\
& +z\left(\frac{z^{n}-\lambda}{\lambda}\right) Q^{\prime}(z)+o\left(r_{1}^{n}\right) .
\end{aligned}
$$

(ii) If $K$ is a compact subset of $\mathbb{T}$ with no isolated points, then

$$
\begin{aligned}
\lim _{n \rightarrow \infty} \| & n \Delta_{n}(F(z), z) \|_{\infty, K} \\
& =\max _{z \in K, \beta \in \mathbb{T}}\left|(\beta-1)\left(P^{\prime}(z)+\beta Q^{\prime}(z)\right)\right| .
\end{aligned}
$$

Proof. (i) It is a straightforward from previous lemmas.

To prove (ii) use the same technique as in Theorem 4.

\section{Numerical Tests}

In this section we present some numerical experiments concerning the main results in Sections 3 and 4.
Theorem 10 ensures that under appropriate assumptions,

$$
\frac{\left\|n \Delta_{n}(F(z), z)\right\|_{\infty, K}}{\max _{z \in K, \beta \in \mathbb{T}}\left|(\beta-1)\left(P^{\prime}(z)+\beta Q^{\prime}(z)\right)\right|} \longrightarrow 1 .
$$

Moreover from the proofs of Lemmas 8 and 9 we can predict where this limit can be observed. In fact near $z_{0}$, when $\left(z_{0}, \beta_{0}\right)$ is the point where the maximum of $\max _{z \in K, \beta \in \mathbb{T}} \mid(\beta-1)\left(P^{\prime}(z)+\right.$ $\left.\beta Q^{\prime}(z)\right) \mid$ is attained, we would observe the convergence.

A second interesting point is that when the maximum $\max _{z \in \mathbb{T}, \beta \in \mathbb{T}}\left|(\beta-1)\left(P^{\prime}(z)+\beta Q^{\prime}(z)\right)\right|$ is attained at a unique point, then for a compact set $K$ with $z_{0} \notin K$ :

$$
\frac{\left\|n \Delta_{n}(F(z), z)\right\|_{\infty, K}}{\max _{z \in \mathbb{T}, \beta \in \mathbb{T}}\left|(\beta-1)\left(P^{\prime}(z)+\beta Q^{\prime}(z)\right)\right|} \longrightarrow l<1 .
$$

We have developed some numerical examples to see these phenomena about Theorem 10.

Example 11. Let $F(z)$ be $F(z)=P(z)+Q(z)=e^{z}+1 /(z-a)$ with $a \in(0,1)$. It is easy to see that the corresponding maximum with $K=\mathbb{T}$ is attained at $\left(z_{0}, \beta_{0}\right)=(1,-1)$. Furthermore the maximum value is $2\left|P^{\prime}(1)-Q^{\prime}(1)\right|$, and it is unique. For $n=2^{p}, p=4,6,8,10,12,14$ we obtain the corresponding Hermite- Fejér approximants (based on the $n$ roots of 1 ) the corresponding $\Delta_{n}(F(z), z)$, and we evaluate the quotient

$$
\frac{\left|n \Delta_{n}(F(z), z)\right|}{\left|P^{\prime}(1)-Q^{\prime}(1)\right|}
$$

in 5000 random points of the $\operatorname{arc} K_{1}=\left[e^{(\pi / 6) \imath}, e^{(\pi / 2) \imath}\right] \subset \mathbb{T}$. As we have said the maximum of the quotients must converge to a value less than 2 . As a second part of the example we evaluate the quotients in 1000 random points of the $\operatorname{arc} K_{2}=$ $\left[e^{0 \imath}, e^{(2 \pi / n) \imath}\right] \subset \mathbb{T}$. This second sequence must converge to 2 ; notice that the great number of evaluations gives an estimate of the supremum norm. 
TABLE 3

\begin{tabular}{cccc}
\hline$p$ & $n$ & Max detected in $K_{1}$ of $\left|n \Delta_{n}(F(z), z)\right| /\left|1.005 F^{\prime}(1.005)\right|$ & Max detected in $K_{2}$ of $\left|n \Delta_{n}(F(z), z)\right| /\left|1.005 F^{\prime}(1.005)\right|$ \\
\hline 4 & 16 & 1.88649 & 1.35356 \\
6 & 64 & 1.67554 & 1.72464 \\
8 & 256 & 1.25126 & 1.27855 \\
10 & 1024 & 0.9862 & 1.0064 \\
12 & 4096 & 0.980863 & 1. \\
14 & 16384 & 0.980855 & 1. \\
\hline
\end{tabular}

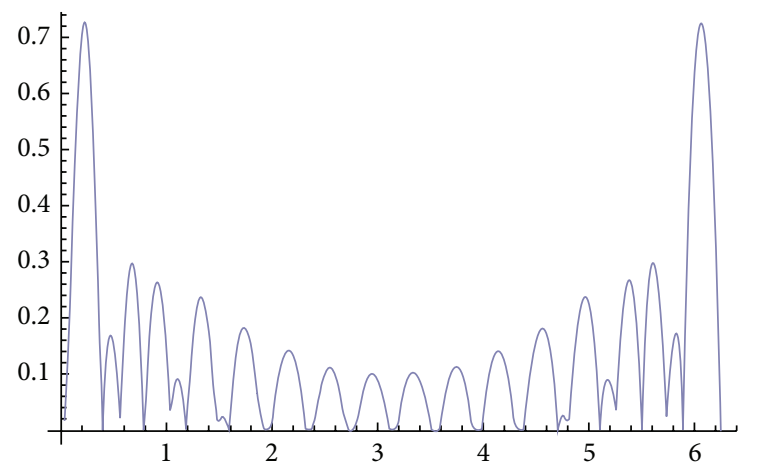

FIGURE 1

Table 1 shows the results observed for $a=0.5$.

Figure 1 shows the graphic of $\left|\Delta_{n}\left(F(z), e^{\imath x}\right)\right|$ for $a=.5$, $n=16$, and $x \in[0,2 \pi]$. In this graphic we can see given in thw following we can see that the maximum of the error is attained near to $x_{1}=0$ or near $x_{1}=2 \pi$ and its corresponding points in $\mathbb{T}$ are near to $z=1$.

Table 2 shows the results observed for $a=0.01$.

Next we are going to apply Theorem 6 . This result and the details of its proof claim that for an analytic function $F(z)$, a compact $\operatorname{arc} K$ of radius $\rho>1$ and under the corresponding assumptions,

$$
\frac{\left\|n \Delta_{n}(F(z), z)\right\|_{\infty, K}}{\max _{z \in K}\left|z F^{\prime}(z)\right|} \longrightarrow 1
$$

and the convergence can be increasing or decreasing; really it depends on the sign of $\left|1-1 / z^{n}\right|-1$.

We must point out that outside the unit disc the algorithms for Hermite-Fejér interpolation can be unstable, so we deal with a compact set near $\mathbb{T}$.

Example 12. Let $F(z)$ be $F(z)=e^{z}$, and let $K, K_{1}$, and $K_{2}$ be the $\operatorname{arcs}\left[1.005,1.005 e^{\imath(\pi / 4)}\right],\left[1.005 e^{\imath(\pi / 16)}, 1.005 e^{l(\pi / 4)}\right]$, and $\left[1.005,1.005 e^{\imath(\pi / 32)}\right]$, respectively. It is easy to see that $\max _{z \in K}\left|z F^{\prime}(z)\right|$ is attained at 1.005 . So we can observe $\left\|n \Delta_{n}(F(z), z)\right\|_{\infty, K_{2}} / \max _{z \in K}\left|z F^{\prime}(z)\right|$ tending to 1 and $\left\|n \Delta_{n}(F(z), z)\right\|_{\infty, K_{1}} / \max _{z \in K}\left|z F^{\prime}(z)\right|$ tending to a number $l<1$. For $n=2^{p}, p=4,6,8,10,12,14$ we obtain the corresponding Hermite-Fejér approximants, and the corresponding $\Delta_{n}(F(z), z)$, and we obtain 5000 evaluations for the quotient

$$
\frac{\left|n \Delta_{n}(F(z), z)\right|}{1.005 F_{1}^{\prime}(1.005)}
$$

in random points of the $\operatorname{arc} K_{1}$. As we have said the maximum of quotients must converge to a value less than 1 . As a second part of the example we obtain 1000 evaluations for the quotients in random points of the $\operatorname{arc} K_{2}$, and this second sequence must converge to 1 . Notice that the great number of evaluations gives an estimation of the supremum norm. Table 3 shows the observed results.

\section{Acknowledgments}

The authors want to give thanks to the referee for the valuable suggestions to improve the paper. The research was supported by Ministerio de Educación y Ciencia of Spain under Grant no. MTM2011-22713.

\section{References}

[1] L. Daruis and P. González-Vera, "A note on Hermite-Fejér interpolation for the unit circle," Applied Mathematics Letters, vol. 14, no. 8, pp. 997-1003, 2001.

[2] P. J. Davis, Interpolation and Approximation, Dover Publications, New York, NY, USA, 1975.

[3] J. C. Mason and D. C. Handscomb, Chebyshev Polynomials, Chapman \& Hall/CRC, Boca Raton, Fla, USA, 2003.

[4] T. J. Rivlin, The Chebyshev Polynomials, John Wiley \& Sons, New York, NY, USA, 1974, Pure and Applied Mathematics.

[5] E. Berriochoa, A. Cachafeiro, and J. M. García-Amor, "An extension of Fejér's condition for Hermite interpolation," Complex Analysis and Operator Theory, vol. 6, no. 3, pp. 651-664, 2012.

[6] E. Berriochoa and A. Cachafeiro, "Algorithms for solving Hermite interpolation problems using the fast Fourier transform," Journal of Computational and Applied Mathematics, vol. 235, no. 4, pp. 882-894, 2010.

[7] C. W. Clenshaw, "A note on the summation of Chebyshev series," Mathematical Tables and Other Aids to Computation, vol. 9, pp. 118-120, 1955.

[8] E. Berriochoa, A. Cachafeiro, and E. Martínez Brey, "Some improvements to the Hermite-Fejér interpolation on the circle and bounded interval," Computers \& Mathematics with Applications, vol. 61, no. 4, pp. 1228-1240, 2011. 
[9] J. Szabados and P. Vértesi, Interpolation of Functions, World Scientific Publishing, Teaneck, NJ, Singapore, 1990.

[10] J. Szabados, "Optimal order of convergence of Hermite-Fejér interpolation for general systems of nodes," Acta Scientiarum Mathematicarum, vol. 57, no. 1-4, pp. 463-470, 1993. 


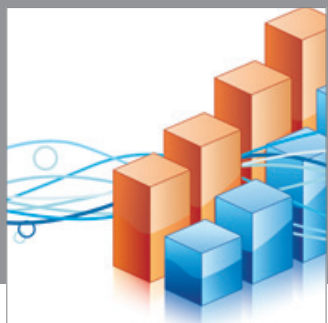

Advances in

Operations Research

mansans

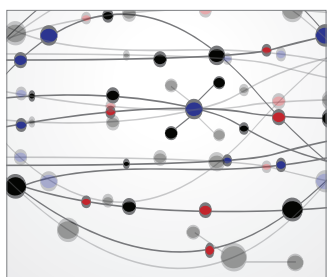

The Scientific World Journal
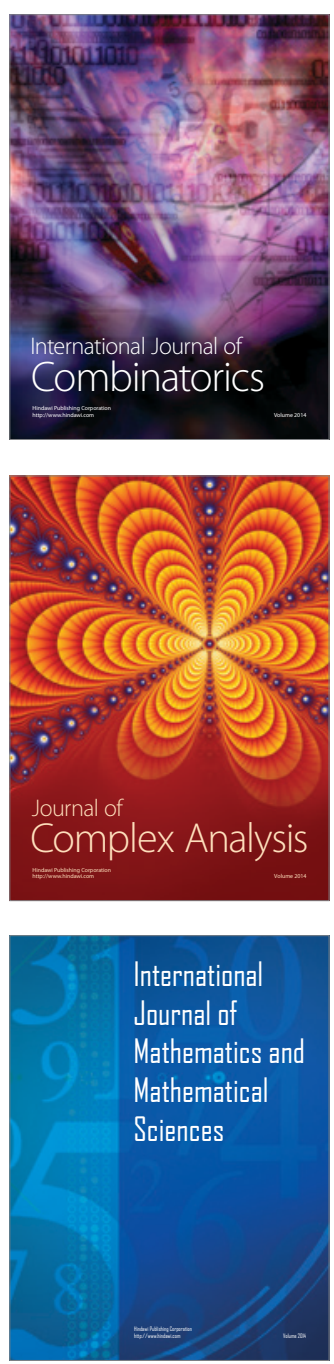
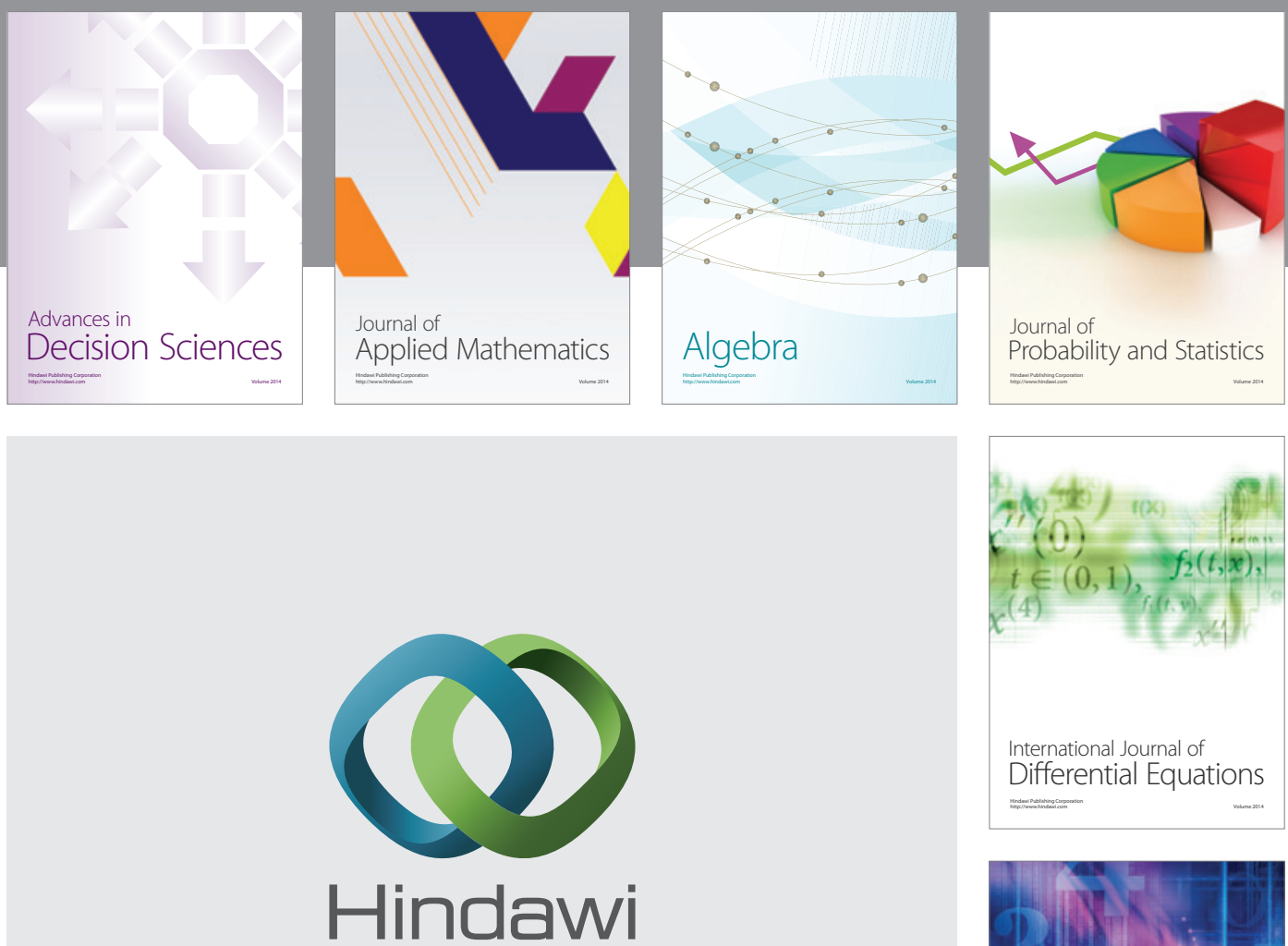

Submit your manuscripts at http://www.hindawi.com
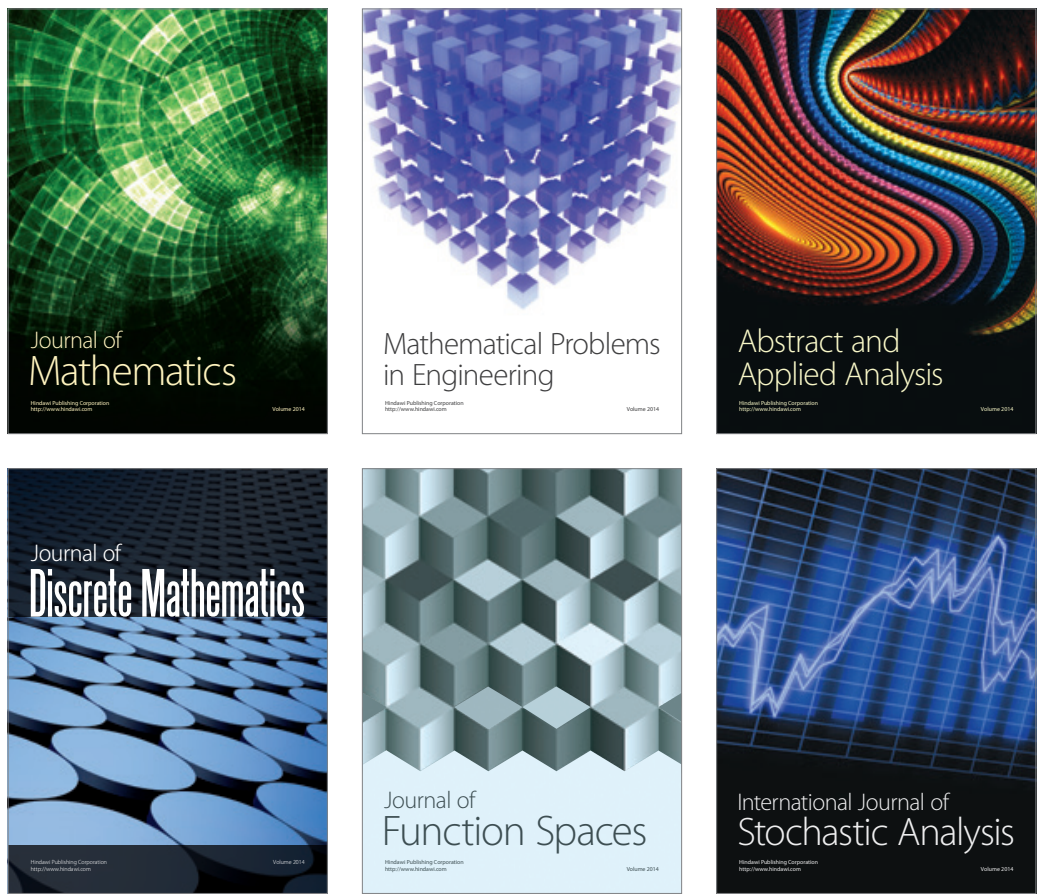

Journal of

Function Spaces

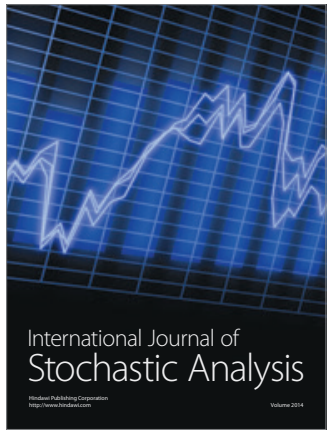

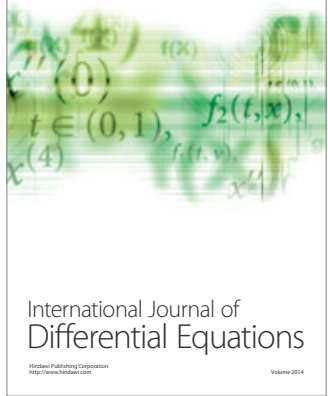
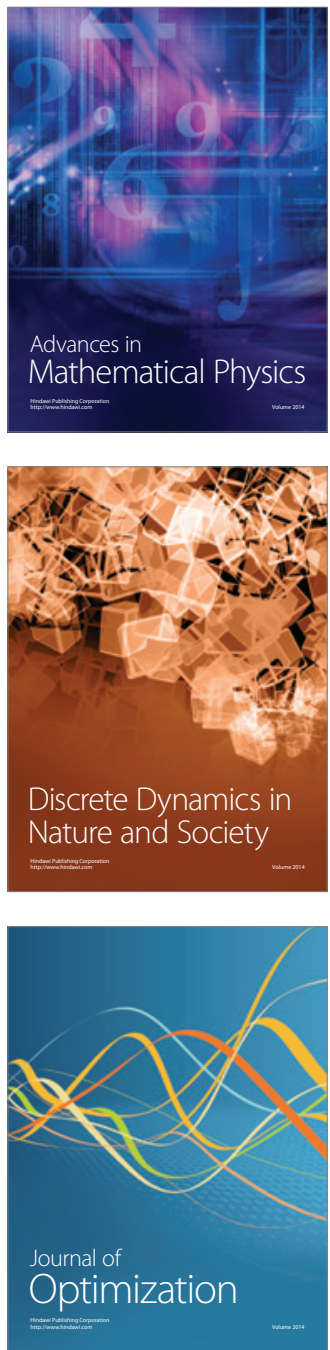